Florida International University FIU Digital Commons

$11-15-2007$

\title{
Intersensory redundancy educates human infants' attention to the prosody of speech
}

Irina Castellanos

Florida International University

DOI: $10.25148 /$ etd.FI14060104

Follow this and additional works at: https://digitalcommons.fiu.edu/etd

Part of the Psychology Commons

\section{Recommended Citation}

Castellanos, Irina, "Intersensory redundancy educates human infants' attention to the prosody of speech" (2007). FIU Electronic Theses and Dissertations. 2072.

https://digitalcommons.fiu.edu/etd/2072

This work is brought to you for free and open access by the University Graduate School at FIU Digital Commons. It has been accepted for inclusion in FIU Electronic Theses and Dissertations by an authorized administrator of FIU Digital Commons. For more information, please contact dcc@fiu.edu. 


\section{FLORIDA INTERNATIONAL UNIVERSITY}

Miami, Florida

INTERSENSORY REDUNDANCY EDUCATES HUMAN INFANTS' ATTENTION TO THE PROSODY OF SPEECH

A thesis submitted in partial fulfillment of the requirements for the degree of MASTER OF SCIENCE in PSYCHOLOGY by

Irina Castellanos 
To: Interim Dean Mark D. Szuchman

College of Arts and Sciences

This thesis, written by Irina Castellanos, and entitled Intersensory Redundancy Educates Human Infants' Attention to the Prosody of Speech, having been approved in respect to style and intellectual content, is referred to you for judgment.

We have read this thesis and recommend that it be approved.

Janat F. Parker

Robert Lickliter

Lorraine E. Bahrick, Major Professor

Date of Defense: November 15, 2007

The thesis of Irina Castellanos is approved.

Interim Dean Mark D. Szuchman

College of Arts and Sciences

Dean George Walker

University Graduate School

Florida International University, 2007 


\section{DEDICATION}

Dedico esta tesis a mis padres y hermano. Sin su amor, apoyo, y paciencia, la terminación de este trabajo no hubiera sido posible. 


\section{ACKNOWLEDGMENTS}

I wish to thank all the members of my committee for supporting my efforts, fostering ideas, and providing insightful suggestions. I would like to express gratitude to my mentor, Dr. Lorraine E. Bahrick, for her generosity of time and for guiding me with dedication and support. I also wish to thank Melissa Shuman and Mariana VaillantMolina for their hard work and encouragement. 
ABSTRACT OF THE THESIS

INTERSENSORY REDUNDANCY EDUCATES HUMAN INFANTS' ATTENTION TO

THE PROSODY OF SPEECH

by

Irina Castellanos

Florida International University, 2007

Miami, Florida

Professor Lorraine E. Bahrick, Major Professor

The following study examined how young infants learn to detect the amodal properties available in prosodic speech (e.g., affect, duration, patterns consisting of tempo, rhythm, and intensity changes) in contexts where intersensory redundancy is not available. It is proposed that the detection of amodal properties in redundant audiovisual stimulation can "educate" selective attention (Gibson, 1979), to those same properties in subsequent nonredundant stimulation (Lickliter, Bahrick, \& Markham, 2006). If so, then infants pre-exposed to redundant audiovisual as compared with nonredundant unimodal auditory speech should discriminate amodal properties of prosodic speech during nonredundant unimodal auditory habituation and testing sessions. Results confirmed predictions and support the hypothesis that, during early development, sensitivity to the amodal properties available in prosodic speech emerges in the context of intersensory redundancy and is later extended to contexts where intersensory redundancy is not available. 
I. THE ROLE OF PROSODY AS A FORM OF COMMUNICATION ….................

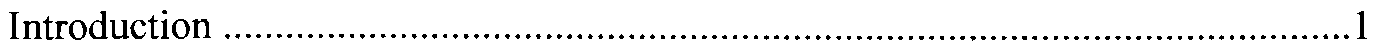

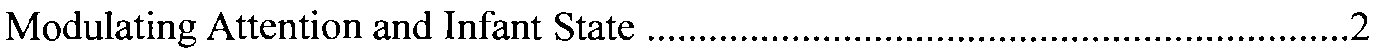

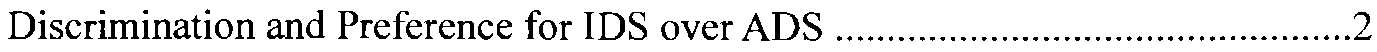

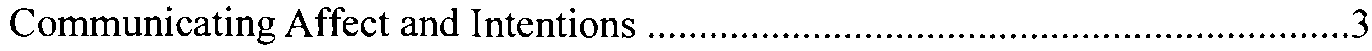

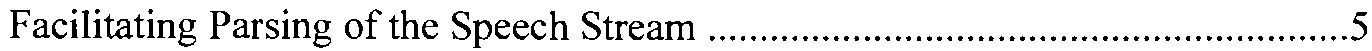

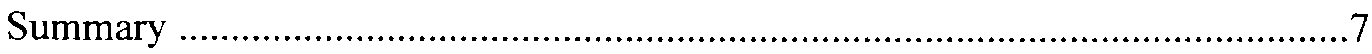

II. THEORIES OF ATTENTION AND PERCEPTION ..............................................

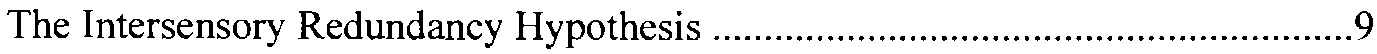

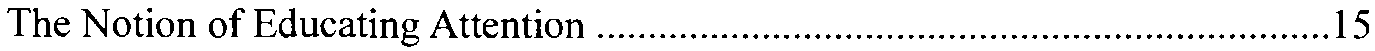

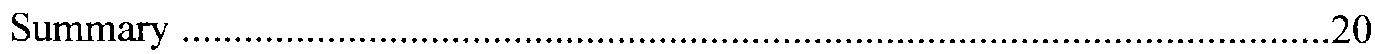

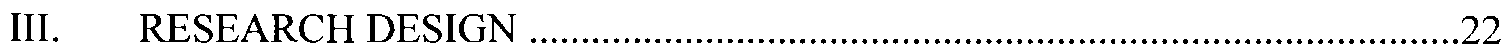

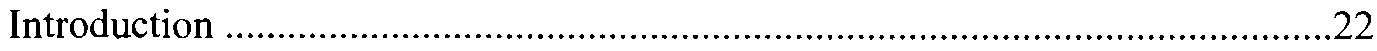

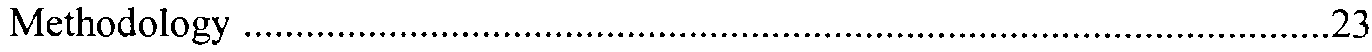

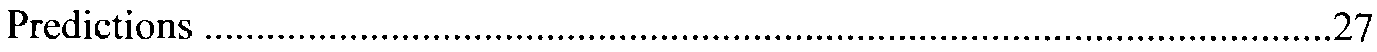

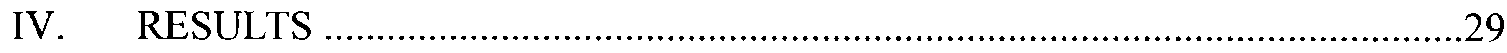

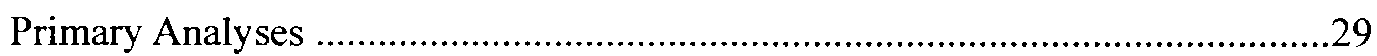

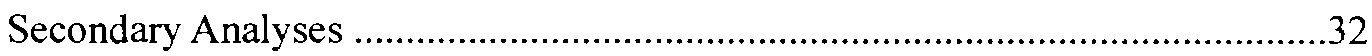

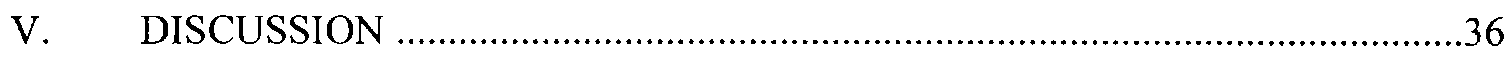

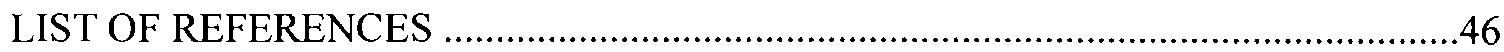




\section{Chapter I}

The Role of Prosody as a Form of Communication

"I have elsewhere maintained that before man used articulate language, he uttered notes in a true musical scale as does the anthropoid ape Hylobates... Before he was a year old, he understood intonations and gestures." C. Darwin (1877).

\section{Introduction}

Infant-directed speech highlights prosodic contours by exaggerating the tempo, rhythm, and pitch of speech over time. Research indicates that across languages adults use infant-directed speech, suggesting that these prosodic modifications function as cross-linguistic universals (Fernald, Taeschner, Dunn, \& Papousek, 1989; Grieser \& Kuhl, 1988; Papousek, Papousek, \& Symmes, 1991). Fernald (1984) has postulated and research has shown that the exaggerated prosodic contours characteristic of infantdirected speech are important for modulating infants' attention and state (Fernald, 1984; Stern, Spieker, Barnett, \& Mackain, 1983; Stern, Spieker, \& MacKain,1982), conveying affect and intention in speech (Fernald, 1989; Fernald, 1993; Fernald \& Kuhl, 1987; Moore, Spence, \& Katz, 1997; Papousek, Bornstein, Nuzzo, Papousek, \& Symmes, 1990; Spence \& Moore, 2003; Trainor, Austin, \& Desjardins, 2000; Werker \& McLeod, 1989) aiding infants in parsing the speech stream and facilitating learning of sound-meaning relations (Christophe, Gout, Peperkamp, \& Morgan, 2003; Fernald \& Mazzie, 1991; Gerkin \& Aslin, 2005; Hirsh-Pasek et al., 1987; Jusczyk, Hirsch-Pasek, Kemler Nelson, \& Kennedy, 1992; Mandel, Kemler Nelson, \& Jusczyk, 1996; Morgan, 1996; Nazzi, Kemler Nelson, Jusczyk, \& Jusczyk, 2000; Shafer, Shucard, \& Jaeger, 1999). 
Research has shown that the prosodic contours characteristic of infant-directed speech (IDS) are used differentially to regulate infants' attention and maintain social interactions (Fernald, 1984; Stern et al., 1982). Mothers use rising prosodic contours to engage infants' attention and once attention has been elicited, they use bell-shaped risefall prosodic contours to maintain infants' interest (Stern et al., 1983). Moreover, mothers use prosody differentially in response to infants' affective states. For example, mothers use falling prosodic contours to comfort infants when they are distressed (Fernald, 1984; Stern et al., 1983). Taken together, these studies suggest that the prosodic contours characteristic of infant-directed speech serve multiple regulating purposes and that mothers can effectively tailor their prosody to individual infant states.

Discrimination and Preference for IDS over ADS

The exaggerated prosodic contours characteristic of infant-directed speech (IDS) are of great interest to young infants and they engage their attention more than the prosodic contours characteristic of adult-directed speech (ADS). Infants as young as two-days old have been shown to look longer at a checkerboard pattern when it produced infant-directed speech over adult-directed speech (Cooper \& Aslin, 1990). In a study where the speech was filtered so that only the prosodic contours could be heard, infants preferred to listen to the prosodic contours of filtered infant-directed speech over the prosodic contours of filtered adult-directed speech (Fernald \& Kuhl, 1987). In an operant preference paradigm, infants can be trained to turn their head to one of two screens so as to activate a particular auditory recording. Using this paradigm, infants have been shown 
to prefer to listen to the prosodic contours of natural speech samples of infant-directed over adult-directed speech (Fernald, 1985). Moreover, when dynamic moving stimuli are used, infants also prefer to listen and view an actor who is speaking in infant-directed speech over adult-directed speech (Werker $\&$ McLeod, 1989). These studies suggest that infants prefer the prosodic contours characteristic of infant-directed speech over adultdirected speech across several behavioral paradigms.

\section{Communicating Affect and Intentions}

The exaggerated prosodic contours characteristic of infant-directed speech have been postulated to communicate affect and intentions (Fernald, 1989; Fernald \& Kuhl, 1987). Researchers have suggested that the pitch variability and range of prosodic contours characteristic of infant-directed speech enhance the vocal expression of emotion which, in turn, contributes to perceivers' discrimination of emotional expressions (Fernald \& Kuhl, 1987; Trainor et al., 2000). The pitch characteristics of infant-directed speech typically range from $90 \mathrm{~Hz}$ to $800 \mathrm{~Hz}$, whereas the pitch characteristics of adultdirected speech typically range from $90 \mathrm{~Hz}$ to $300 \mathrm{~Hz}$ (Fernald, 1985). Phrases conveying approval and prohibition spoken in infant-directed speech vary greatly in their prosodic contours. Rise-fall pitch contours are typical of phrases conveying approval such as "Oh, good girl!" In contrast, low mean pitch, narrow pitch range with sharp staccato contours are typical of phrases conveying prohibition such as "No, no, no, don't touch!" (Fernald, 1989; Fernald \& Kulh, 1987).

Research suggests that infants and adults perceive affect and communicative intent on the bases of prosodic contours characteristic of infant-directed speech. Fernald 
(1989) demonstrated that adults (both naïve and experienced perceivers of infant-directed speech) can significantly identify the communicative intent (approval, prohibition, attention-bid, comfort, and game/telephone) of a speaker based solely on prosodic contours when samples were spoken in infant-directed speech as compared with when spoken in adult-directed speech. For example, the communicative intent "approval," was correctly identified by fifty-three out of eighty adult subjects when the utterance was heard as filtered infant-directed speech; however, when the utterance was heard as filtered adult-directed speech only two out of eighty adult subjects correctly identified the category.

Infants of four to nine months of age have been shown to respond with significantly more positive affect while hearing infant-directed speech than when hearing adult-directed speech (Werker \& McLeod, 1989). Five-month-old infants respond with significantly more positive affect while hearing infant-directed phrases conveying approval than when hearing infant-directed phrases conveying prohibition. Infants also respond with significantly more negative affect while hearing infant-directed phrases conveying prohibition than when hearing infant-directed phrases conveying approval (Fernald, 1993). Similarly, four-month-old infants prefer to listen to infant-directed speech when it specifies approval than when it specifies prohibition (Papousek et al., 1990). Research has shown that four-month-old infants are able to categorize natural, unfiltered infant-directed passages conveying approval versus prohibition (Bahrick et al., 2007; Castellanos, Shuman, \& Bahrick, 2004), suggesting that IDS conveys intention to young infants. Furthermore, infants of six- but not four-months of age are able to 
categorize infant-directed utterances conveying approval versus comfort based solely on non-linguistic prosodic features such as variations in frequency, rhythm, and intensity in stimuli (Moore et al., 1997; Spence \& Moore, 2003). These studies demonstrate that the prosodic contours characteristic of infant-directed speech over adult-directed speech allow affect and intention to be more accessible to both infants and adults.

\section{Facilitating Parsing of the Speech Stream}

The slower tempo, elongated pauses, exaggerated vowel length, and the frequent repetition characteristic of infant-directed speech are thought to contribute to infants' development of language acquisition and comprehension (Hirsh-Pasek et al., 1987; Morgan, 1996). Behavioral and physiological paradigms have shown that the prosodic contours characteristic of infant-directed speech are so dramatic and highlighting (contain exaggerated tempo, rhythm, and pitch changes over time) compared to adult-directed speech that they facilitate infants' ability to parse the speech stream (Nazzi et al., 2000; Shafer et al., 1999; Morgan, 1996).

Parsing the speech stream is defined as the ability to abstract holistic units (i.e. specific words) from continuous speech. Parsing continuous speech into units is considerably difficult for naïve perceivers because pauses do not reliably separate individual words (Christophe et al., 2003; Gerken \& Aslin, 2005). Thus, naïve perceivers often are unable to rely on pauses as signals for when one word ends and the next begins. Naïve perceivers can rely, however, on prosody instead of pauses to parse continuous speech into units. Infants are highly sensitive to the pitch changes and frequent pauses characteristic of infant-directed speech that mark boundaries of prosodic units 
(Christophe et al., 2003). For example, infants of four and a half-months-of age are able to discriminate and react to disruptions of normal prosodic boundaries (Hirsch-Pasek et al., 1987; Jusczyk et al., 1992). Two-month-old infants increase their visual fixation to a change in word order more so when they listen to sentences that are spoken using natural prosody to link the words of the sentence together (causing words to be prosodically linked) as compared to when the same words of the sentence were simply listed one after the other and as compared to when they listen to sentence fragments, concatenated from two separate sentences, depicting incoherent prosody (Mandel et al., 1996). For example, the individual words contained in a sentence conveying approval such as "What a wonderful job!" are linked by prosodic patterns specifying approval. However, the individual words contained in the sentence may also be read in a monotonous fashion as if reading a grocery list. This study provides evidence suggesting that young infants can abstract holistic units or words from a stream of continuous speech when words are prosodically linked.

The methods by which parents introduce novel sound-meaning relations also contribute to speech parsing and language acquisition. Research has shown that when engaging with their infants, mothers introduce new labels for unfamiliar objects at the peaks of their prosodic contours (Fernald \& Mazzie, 1991). For example, the label "socks" was presented at an increasingly high pitch peak when speaking the phrase "Then he put on his yellow socks." This action facilitates infants' perceptual and attentional development by highlighting words of importance within the stream of continuous speech. Parents also use synchrony between labeling an object using infant- 
directed speech and showing an object to help infants learn novel sound-referent relations (Gogate, Bahrick, \& Watson, 2000) and research indicates that infants benefit from this synchrony (Gogate \& Bahrick, 1998).

\section{Summary}

The studies reviewed in this chapter indicate that the development of infants' ability to detect changes in prosodic speech patterning is a prerequisite for understanding their caregiver's intent and signals. Little previous research, however, has investigated how multimodal speech can aid in infants' ability to detect changes in prosodic patterning. As noted in this chapter, research on infant-directed speech has primarily been conducted on infants' perception of unimodal auditory speech (e.g., Moore, et al., 1997; Spence \& Moore, 2003). However, speech is typically multimodal and involves a speaker who uses gestures and facial movements that are coordinated with their speech sounds. Prior research on audiovisual perception has demonstrated that intersensory redundancy facilitates the detection of amodal properties (Bahrick, Flom, \& Lickliter, 2002; Bahrick \& Lickliter, 2000, 2002; Bahrick et al., 2007; Castellanos et al., 2004; Castellanos, Vaillant-Molina, Lickliter, \& Bahrick, 2006; Flom \& Bahrick, 2007; Lickliter, Bahrick, \& Honeycutt, 2002, 2004; Lickliter, et al., 2006) more so than when intersensory redundancy is not available. Intersensory redundancy refers to the spatially coordinated and temporally synchronous presentation of invariant information specifying an event across multiple sensory modalities. However, stimulation that is redundant across the auditory and visual sensory modalities is not always available in naturalistic contexts. For example, no intermodal redundancy for amodal properties in speech is 
available when speech is heard from a nearly room or when the speaker's face is not visible. Research has not yet investigated the process by which amodal properties available in speech can be detected in contexts where intersensory redundancy is not available. 


\section{Chapter II}

Theories of Attention and Perception

The Intersensory Redundancy Hypothesis

According to the intersensory redundancy hypothesis (IRH), in early

development, information presented redundantly and in temporal synchrony across two or more sensory modalities recruits attention and facilitates perceptual learning of amodal properties more successfully than when the same information is presented to only one sensory modality (Bahrick et al., 2002; Bahrick \& Lickliter, 2000, 2002). Amodal properties are properties which can be perceived redundantly or invariantly across several sensory modalities (i.e., are bimodally or multimodally specified). Tempo, for example, is an amodal property because it offers redundant or invariant information to the visual, auditory, and tactile sensory modalities. Multimodal presentations provide better information for amodal properties such as temporal synchrony (Bahrick, 1983, 1987, 1988, 2001), intensity, prosody (Bahrick et al., 2007; Castellanos et al., 2004), affect (Flom \& Bahrick, 2007), tempo (Bahrick et al., 2002; Castellanos et al., 2006), and rhythm (Bahrick \& Lickliter, 2000) than nonredundant presentations. These amodal properties are available in audiovisual speech and are important for discriminating meaning in speech. In contrast, information presented to only one sensory modality or nonredundantly (temporal asynchrony) across several sensory modalities recruits attention to modality specific properties more successfully than when the same information is presented redundantly to multiple sensory modalities (Bahrick, Lickliter, \& Flom, 2006). Modality specific properties are properties which can only be specified 
in one sensory modality. The orientation of a pattern, for example, is a modality specific property because it offers information to only the visual sensory modality. Nonredundant (unimodal or temporally asynchronous) presentations provide better information for modality-specific properties such as orientation (Bahrick et al., 2006), color (VaillantMolina, Gutierrez, Bahrick, 2005), pitch, and timbre than redundant presentations. Numerous studies indicate that infants, from an early age, are extremely sensitive to temporal synchrony between auditory and visual stimulation (Bahrick, 1983, 1987, 1988, 2001; Lewkowicz, 1992, 1996; Walker-Andrews, 1997). For example, Bahrick (2001) found that four-week-old infants detect temporal synchrony. In the study, infants were habituated to redundant, temporally synchronized audiovisual displays of an alternating sequence of single and compound objects striking a surface. Results indicate that infants displayed a significant increase in visual fixation when tested on nonredundant, temporally asynchronous audiovisual displays of those same stimulus objects. Pickens, Field, Nawrocki, \& Martinez (1994) found that infants as young as three-months-old fixate significantly longer to video displays in which dynamic moving faces are synchronized to a speaker's voice versus video displays of faces that are not synchronized to a speaker's voice. Temporal synchrony can also aid infants in segmenting the speech stream. Hollick, Newman, \& Jusczyk (2005), investigated infants' ability to parse and display memory for target words contained in a single speech stream when two streams of speech were played concurrently. They presented sevenand-a-half-month-old infants a visual display (a dynamic face, or a static face, or an oscilloscope) with two voices of equal loudness (a female voice speaking in infant- 
directed speech and a distractor male voice speaking in adult-directed speech). However, only one of the voices (the female voice) was played in temporal synchrony with the visual display (the dynamic face and oscilloscope in Experiment 1 and 4, respectively). Infants in Experiment 1 and 4 who were presented with a visual display depicting a dynamic face or an oscilloscope moving in temporal synchrony with one of the two voices demonstrated significantly longer looking to the target words spoken in synchrony with the display than to unfamiliar words. However, infants in Experiment 2 who were presented with a visual display depicting a dynamic face speaking out of temporal synchrony with two voices (no intersensory redundancy provided) did not demonstrate significant longer looking to the target words than to the unfamiliar words, showing no learning. Furthermore, infants in Experiment 3 who were presented with a visual display depicting a static face presented with two voices (no intersensory redundancy) also showed no learning. Taken together, these findings indicate that, on the basis of temporal synchrony between visual and auditory information, infants were able to selectively attend to the synchronized female voice while ignoring the asynchronized or distractor male voice, were able to parse target words from the synchronized stream of speech and learn to discriminate them from novel words.

A recent study (Bahrick et al., 2007) tested predictions of the IRH in the domain of speech perception. We assessed whether four-month-old infants could perceive a change in meaning from passages conveying prohibition to passages conveying approval or vice versa when presented with redundant audiovisual speech versus when presented with unimodal auditory or asynchronous audiovisual speech. Audiovisual speech 
presentations offer intersensory redundancy in the form of temporal synchrony, highlighting common tempo, rhythm, and intensity shifts between the auditory and visual sensory modalities. In contrast, unimodal auditory and asynchronous audiovisual speech presentations provide no intersensory redundancy and do not highlight tempo, rhythm, and intensity shifts across the sensory modalities. Unimodal auditory speech presentations do not offer intersensory redundancy because they do not provide stimulation to the other sensory modalities. Similarly, asynchronous audiovisual speech presentations do not offer intersensory redundancy because such presentations eliminate the temporal synchrony between the auditory and visual sensory modalities. Therefore, it was predicted that detection of meaning across speech passages would be facilitated by redundant audiovisual speech presentations and attenuated by both nonredundant presentations (unimodal auditory and asynchronous audiovisual speech) if intersensory redundancy was important for early prosody perception. We habituated infants to displays of one of two actresses reciting passages in one of two prosodic patterns (approval vs. prohibition) under either redundant audiovisual, unimodal auditory or asynchronous audiovisual conditions. Infants in the redundant audiovisual condition were presented with a dynamic moving face that produced natural and synchronous speech sounds; those in unimodal auditory condition were presented with still images of the actress' face while concurrently hearing the spoken passages. Infants in the asynchronous audiovisual condition were presented with temporally asynchronous information, in that the dynamic face and the spoken passages were out of synchrony with respect to one another. The asynchrony was achieved by presenting the visual 
information approximately three-seconds before the onset of the auditory information. After habituation, infants were randomly assigned to receive two test trials depicting either a novel passage read in a novel prosody or a novel passage read in the familiar prosody. Results indicated that infants who received redundant audiovisual stimulation significantly discriminated the changes in prosody, whereas infants who received unimodal auditory stimulation or asynchronous audiovisual stimulation did not show significant evidence of discrimination.

The findings support the intersensory redundancy hypothesis and demonstrate that four-month-old infants are able to discriminate changes in prosodic patterns conveying approval and prohibition in the presence of redundancy, provided by a synchronously moving face, but not in its absence. Furthermore, these findings provide evidence against the notion that infants selectively attend more to redundant audiovisual than to unimodal auditory presentations simply because redundant audiovisual presentations offer more information about an event. Infants in the asynchronous audiovisual condition were provided with the same amount and type of stimulation (received both the auditory and visual information) as infants in the redundant audiovisual condition, but were unable to discriminate a change in prosody. The failure to discriminate the change in prosody is likely due to the lack of intersensory redundancy, i.e., the disruption of temporal synchrony between the visual and auditory displays. These results suggest that synchronous audiovisual presentations provide better information for amodal properties such as affect, duration, patterns consisting of tempo, rhythm and intensity changes in 
speech, which are important for distinguishing between prosodic patterns conveying approval and prohibition.

Comparative studies with avian species indicate that precocial quail embryos are also highly sensitive to intersensory redundancy in the form of temporal synchrony (Lickliter et al., 2002, 2004). These studies assessed quail embryos' perceptual learning and memory of a maternal quail call comprised of amodal temporal properties. It was predicted that intersensory redundancy in the form of temporal synchrony between visual and auditory stimulation would highlight the amodal properties (e.g., tempo, rhythm, duration) available in maternal quail calls more so than when intersensory redundancy was disrupted. In the Lickliter et al. (2002) study, quail embryos received prenatal exposure for $10 \mathrm{~min} / \mathrm{hr}$ for 6,12 , or $24 \mathrm{hrs}$ prior to hatching to either nonredundant stimulation (unimodal auditory or asynchronous audiovisual stimulation) or redundant audiovisual stimulation. Quail embryos in the unimodal auditory condition were prenatally presented with only auditory information specifying the maternal quail call; those embryos in the asynchronous audiovisual condition were prenatally presented with a light that flashed out of temporal synchrony with the notes of the maternal quail call, while those in the redundant audiovisual condition were prenatally presented with intersensory redundancy in the form of a pulsing light that flashed in temporal synchrony (at the same tempo, rhythm, and duration) with the notes of the maternal quail call. Twenty-four hours following hatching, quail chicks were tested in a circular arena to determine their latency and approach to the familiar versus novel maternal quail call. Quail chicks who received prenatal redundant audiovisual exposure demonstrated 
perceptual learning postnatally under all exposure periods $(10 \mathrm{~min} / \mathrm{hr}$ for 6,12 , and 24 hrs). However, chicks who received prenatal unimodal auditory exposure demonstrated perceptual learning postnatally only following the longest period of prenatal exposure (10 $\mathrm{min} / \mathrm{hr}$ for $24 \mathrm{hrs}$ ). When prenatal exposure time was considered, quail chicks who received redundant audiovisual stimulation learned the familiar maternal call four times faster than those chicks who received unimodal auditory stimulation. Quail chicks who received asynchronous audiovisual stimulation, however, did not show evidence of learning the maternal quail call under any prenatal exposure period. These results provide added support for the hypothesis that redundant audiovisual stimulation highlights the amodal properties found in maternal quail calls and facilitates learning and memory of those properties in early development.

Together, human and animal studies suggest that redundant (synchronous) audiovisual contexts highlight amodal properties such as tempo, rhythm and duration more so than nonredundant contexts (e.g., those contexts that offer no audiovisual synchrony including unimodal auditory and asynchronous audiovisual stimulation).

\section{The Notion of Educating Attention}

Research has shown that during early development infants are best able to perceive amodal properties with the benefit of intersensory redundancy (Bahrick et al., 2002; Bahrick \& Lickliter, 2000, 2002; Bahrick et al., 2007; Flom \& Bahrick, 2007; Lickliter et al., 2002, 2004). How then do infants learn to detect amodal properties in contexts where intersensory redundancy is not available? The notion of educating attention serves as one possible answer to this question. Lickliter et al. (2006) postulate 
that the detection of amodal properties in redundant audiovisual stimulation can scaffold or highlight selective attention to those same stimulus properties in subsequent nonredundant stimulation. For example, once infants detect rhythm, an amodal property, in redundant audiovisual stimulation, it is predicted that the property should be highlighted and attended to in subsequent nonredundant stimulation.

The notion of educating attention is similar to stimulus priming (Schacter, 1987; Schacter \& Badgaiyan, 2001; Tulving \& Schacter, 1990). Stimulus priming refers to the transient effects of prior exposure (usually 30 milliseconds) to stimulus properties on subsequent behavior or performance (Schacter, 1987; Tulving \& Schacter, 1990). Classic examples of stimulus priming include the ability to identity or complete words following exposure to target words. For example, participants can be primed to a list of target words specifying fruit such as pineapple and mango, and asked afterwards to complete the three letter stem (FRU). Based on stimulus priming theory, participants who receive priming, as compared to those who do not receive priming, should be more likely to complete the letter stem with the word FRUIT than with words like FRUGAL or FRUMPY which do not hold commonalities with the target words.

Stimulus priming most often occurs on an unconscious level and is described as an automatic process (Schacter \& Badgaiyan, 2001; Tulving \& Schacter, 1990). For example, studies have identified a subset of amnesic patients who can exhibit effects of priming (Hamann, Squire, \& Schacter, 1995). This suggests that priming effects can occur independently of possible impairments in explicit memory. In contrast, the notion of educating attention involves selective attention to stimulus properties highlighted as a 
result of perceptual or conceptual learning. Subsequent behavior can be a function of either unconscious or conscious selective attention. Since educating attention can occur as a function of conscious attention it can promote long lasting effects, whereas priming has only transient effects.

Bahrick \& Lickliter (2000) indirectly examined the notion of educating attention. The authors investigated five-month-old infants' ability to discriminate rhythm, an amodal property, under redundant audiovisual and unimodal visual conditions. Infants in the redundant audiovisual condition were habituated to a dynamic image of a toy hammer tapping a particular rhythm. Intersensory redundancy was provided by presenting the auditory and visual stimulation in temporal synchrony. Infants in the unimodal visual condition, however, were habituated to a dynamic visual image of a hammer tapping a particular rhythm without its accompanying soundtrack. Following habituation, all infants received unimodal visual test trials depicting a change in rhythm. Results demonstrated that infants were able to discriminate a change in rhythm when they were habituated to redundant audiovisual displays and tested with unimodal visual displays. However, infants who were habituated to and tested with unimodal visual displays did not detect a change in rhythm. These findings suggest that, during early development, previous experience with redundant audiovisual stimulation can serve to highlight the amodal property of rhythm in subsequent nonredundant stimulation.

Lickliter et al. (2006) directly investigated the notion of educating attention to amodal properties with quail chicks. This study investigated if a short prenatal exposure to redundant audiovisual stimulation could direct quail embryos attention to the amodal 
properties in a maternal quail call in subsequent nonredundant auditory stimulation and if chicks could display memory for these properties following a two day delay. In the first experiment, embryos were randomly assigned to an experimental condition: a condition where they received $10 \mathrm{~min} / \mathrm{hr}$ for $6 \mathrm{hrs}$ of initial redundant audiovisual stimulation followed by $10 \mathrm{~min} / \mathrm{hr}$ for $18 \mathrm{hrs}$ of unimodal auditory stimulation (redundant $\rightarrow$ unimodal), or one of four control conditions: a condition that controlled for the sequence of stimulation in which embryos received $10 \mathrm{~min} / \mathrm{hr}$ for $18 \mathrm{hrs}$ of initial unimodal auditory stimulation followed by $10 \mathrm{~min} / \mathrm{hr}$ for $6 \mathrm{hrs}$ of redundant audiovisual stimulation (unimodal $\rightarrow$ redundant), a condition that controlled for overall amount and type of stimulation in which embryos received $10 \mathrm{~min} / \mathrm{hr}$ for $6 \mathrm{hrs}$ of initial asynchronous audiovisual stimulation followed by $10 \mathrm{~min} / \mathrm{hr}$ for $18 \mathrm{hrs}$ of unimodal auditory stimulation (asynchronous $\rightarrow$ unimodal), a condition where embryos only received 10 $\mathrm{min} / \mathrm{hr}$ for $18 \mathrm{hrs}$ of unimodal auditory stimulation to examine if unimodal auditory stimulation was sufficient, or a condition where embryos only received $10 \mathrm{~min} / \mathrm{hr}$ for 6 hrs of redundant audiovisual stimulation to examine if redundant audiovisual stimulation was sufficient for the discrimination and learning of amodal properties. Intersensory redundancy was provided by presenting the auditory call in temporal synchrony (same tempo, rhythm, and duration of notes) with a pulsing light. Forty-eight hours following hatching, quail chicks were tested in a circular arena to determine their latency and approach to the familiar versus novel maternal quail call. Results indicate that quail chicks demonstrated learning and memory for the amodal properties available in the 
maternal quail call only when redundant audiovisual stimulation preceded unimodal auditory stimulation (redundant $\rightarrow$ unimodal), and not in any of the other four conditions (including unimodal $\rightarrow$ redundant), suggesting that the order of stimulation mattered. Prenatal redundant audiovisual stimulation afforded quail embryos an opportunity to attend to amodal properties in subsequent unimodal auditory stimulation.

In the second experiment, the delay interval between initial redundant audiovisual stimulation and subsequent unimodal auditory stimulation was manipulated. Embryos in this experiment were randomly assigned to two experimental conditions: conditions where 2 or 4 hrs delays were implemented between initial redundant audiovisual stimulation and subsequent unimodal auditory stimulation, or a control condition: a condition which presented no delays between stimulation (mirrors the experimental condition from Experiment 1). Results indicate that following redundant audiovisual stimulation, quail chicks under all delay conditions of subsequent unimodal auditory stimulation were able to selectively attend to the amodal properties available in maternal quail calls. Temporal synchrony between the visual and auditory stimulation guided discrimination, learning, and memory for the temporal properties of a maternal quail call in subsequent unimodal auditory stimulation, even after delays of 2 and 4 hours.

Taken together, these experiments provide powerful data indicating the beneficial nature of intersensory redundancy for guiding future perception, attention, and memory for amodal properties. Intersensory redundancy in the form of temporal synchrony facilitates attention to amodal properties, provides a foundation to generalize across 
contexts of stimulation and provides greater sustained memory than unimodal auditory stimulation for amodal properties.

A similar study was conducted with human infants to directly investigate the notion of educating attention to the amodal property of tempo (Castellanos, et al., 2006). We investigated whether two-month-old infants' attention could be educated to the tempo of an event (toy hammer tapping) during a unimodal visual habituation session, by presenting redundant audiovisual as compared with unimodal visual pre-exposure trials prior to habituation. We predicted that infants viewing a silent film of a hammer tapping at one of two tempos would be able to discriminate between the tempos if beforehand they received redundant audiovisual pre-exposure to the film and soundtrack of the hammer tapping. In contrast, we predicted that infants would not be able to discriminate between the tempos if beforehand they received unimodal visual pre-exposure to the hammer tapping silently. These predictions were formed given the hypothesis that redundant audiovisual pre-exposure (as compared to unimodal visual pre-exposure) educates attention to amodal properties and thus allows infants to detect amodal properties such as tempo throughout subsequent unimodal presentations. Results demonstrated that only infants who received redundant audiovisual pre-exposure detected the novel tempo, supporting the hypothesis that detection of amodal properties such as tempo in redundant audiovisual stimulation scaffolds attention to those same stimulus properties in subsequent conditions where intersensory redundancy is not available such as unimodal visual stimulation. 
Taken together, the studies presented in this chapter indicate that the presence of intersensory redundancy (temporal synchrony) initially serves to highlight the invariance in temporal patterning across the modalities and results in the discrimination of amodal properties in subsequent nonredundant stimulation. These research findings suggest that both precocial quail infants and human infants demonstrate education of attention and show enhanced processing of amodal properties as a results of redundant audiovisual stimulation.

The following chapter presents a study designed to extend these animal and human based findings to the domain of speech perception. It asks if three-month-old infants' attention can be educated to the amodal properties available in prosodic speech (such as intonation, affect, duration, patterns consisting of tempo, rhythm, and intensity changes) by pre-exposing infants to contexts where intersensory redundancy is available as compared with contexts where redundancy is not available prior to habituation and testing. 


\section{Chapter III}

\section{Research Design}

\section{Introduction}

I hypothesized that infants first learn to perceive meaningful categories in speech, such as classes of phrases specifying approval versus prohibition, in redundant audiovisual stimulation and, as development occurs, they extend their ability to perceive such categories to nonredundant stimulation (see Bahrick et al., 2007). One basis for this learning is through the detection of amodal properties in redundant audiovisual stimulation. Research has demonstrated that infants of four-months-of age are able to discriminate between prosodic speech samples conveying approval versus prohibition across six phrases under redundant audiovisual conditions, but not under nonredundant conditions (unimodal auditory or asynchronous audiovisual) (Bahrick et al., 2007). If intersensory redundancy highlights prosodic speech (comprised of amodal properties such as affect, duration, patterns consisting of tempo, rhythm, and intensity changes), how do infants learn to detect those amodal properties in contexts where redundancy is not available? The following experiment attempted to answer this question by investigating the conditions that enhance infants' learning of prosodic patterns specifying approval and prohibition in nonredundant stimulation. Lickliter et al., (2006) postulate that the detection of amodal properties in redundant audiovisual stimulation can scaffold or "educate" infants' selective attention to those same stimulus properties in subsequent nonredundant stimulation. Consistent with Bahrick et al. (2007), the detection of prosodic speech (comprised of amodal properties) likely first emerges in redundant 
audiovisual conditions and is later extended to nonredundant conditions. Therefore, I propose that once infants detect the amodal properties available in prosodic speech passages in redundant audiovisual stimulation, their attention should be directed to those same stimulus properties in subsequent unimodal auditory stimulation. It was predicted that if prior exposure to redundant audiovisual stimulation directs infants' attention to amodal properties (such as affect, duration, patterns consisting of tempo, rhythm, and intensity changes in speech) in subsequent unimodal auditory stimulation, then infants pre-exposed to redundant audiovisual stimulation as opposed to unimodal auditory stimulation should discriminate changes in prosodic patterns during testing. In essence, it was predicted that the pre-exposure condition (redundant audiovisual vs. unimodal auditory) would differentially effect infants' detection of prosody during contexts where intersensory redundancy was not available.

\section{Methodology}

\section{Participants}

Forty infants (21 males and 19 females) were tested and included in the final sample. They had a mean age of 109 days $(S D=5.11)$. Thirty of the infants were Hispanic, seven were Causasian, two were African American, and one was of Asian origin. All infants were healthy and had no known complications at delivery, had a gestational period of at least 38 weeks, and an APGAR score of 9 or greater. APGAR is an acronym for an exam performed by medical professionals rating infants' appearance, pulse, grimace, activity, and respiration; a score of 9 or greater indicates that the infant is in good health and did not require immediate medical care (see Apgar, 1953, for more 
information). A total of 15 additional infants were tested but their data were not included in the final sample: 6 due to experimenter errors, 4 due to failure to habituate in fewer than 20 trials, 2 due to computer failures, 2 due to excessive fussiness, and 1 due to fatigue (see Procedure for details).

\section{$\underline{\text { Stimuli }}$}

The stimuli consisted of a videotaped recording of two actresses. The films depicted the face and shoulder area of the actresses. One actress was light skinned and had shoulder length light brown hair and the other actress was olive skinned and had long dark brown hair. The redundant audiovisual displays depicted a dynamically moving face producing natural and synchronous infant-directed speech. These displays provided intersensory redundancy by affording the infant with invariant information specifying the affect, duration, tempo, rhythm, and intensity changes of the passages across the visual and auditory sensory modalities. The unimodal auditory displays depicted a static, nonmoving, face in three affective-neutral poses accompanied by the same infant-directed speech used in the redundant audiovisual condition. These displays did not provide audiovisual redundancy because they offered information about the affect, duration, tempo, rhythm, and intensity of speech only through the auditory sensory modality; the visual sensory modality did not receive any information concerning the prosody of speech because the displays were static and affect-neutral. The actresses recited two passages (each comprised of three phrases), using infant-directed speech to convey two different prosodic patterns. Each passage was spoken in prosodic patterns characteristic of approval and prohibition and the corresponding affect was visible. Passage 1 consisted 
of the phrases "Look at you," "Come over here by me," and "Where's the baby going?" Passage 2 consisted of the phrases "You did this," "Gentle with the baby," and "Whose doggy is that?" Each passage contained approximately the same number of syllables (passage 1, $\mathrm{N}=15$; passage 2, $\mathrm{N}=14$ ).

\section{Apparatus}

Infants sat in a standard infant seat facing a color television monitor (Sony KV-20520) approximately $50 \mathrm{~cm}$ away. A black cloth surrounded the television monitor to obscure extraneous stimuli and two apertures allowed trained observers to view the infants' visual fixations. Observers, unaware of the infants' condition, depressed buttons on a joystick recording the length of the infants' visual fixations. The joystick was connected to a computer which collected the data on line. To prevent extraneous noise from distracting the infants and to reduce the time needed for switching tapes from one trial to the next, I used four Panasonic video desks (DS545 and AG7750) to play the stimuli.

\section{Procedure}

Pre-exposure Session: Infants initially participated in a pre-exposure session. Infants were randomly assigned to receive either redundant audiovisual pre-exposure $(n=20)$, or unimodal auditory pre-exposure $(n=20)$. Within these conditions, half of the infants were pre-exposed to passages conveying approval and half were pre-exposed to passages conveying prohibition. The passages (passage 1 vs. passage 2 ) and the actress reciting the passages (actress 1 vs. actress 2) were also counterbalanced across infants. Infants received four 15 -second trials to a video display of an actress reciting a passage 
(consisting of three phrases) spoken in infant-directed speech conveying either approval or prohibition. Infants who received redundant audiovisual pre-exposure were preexposed to a video display depicting a naturalistic, synchronous face and voice speaking the passages using an approving or prohibiting prosody with the appropriate affect. Infants who received unimodal auditory pre-exposure, however, were pre-exposed to a video display depicting a non-moving face in three affective-neutral poses accompanied by the same vocal track speaking the passages using an approving or prohibiting prosody. Habituation Session: After the pre-exposure session was complete, infants participated in an infant-controlled habituation procedure to assess whether the redundant audiovisual vs. unimodal auditory pre-exposure differentially influenced the detection of prosody specifying approval vs. prohibition. There was approximately a one-minute delay between the pre-exposure and habituation sessions $(65.39$ seconds $(S D=29.00)$ for infants in the redundant audiovisual pre-exposure condition and 59.53 seconds $(S D=$ 14.32) for infants in the unimodal auditory pre-exposure condition). All infants were habituated to a unimodal auditory presentation of the same stimuli (familiar infantdirected passage spoken in the familiar approving or prohibiting prosodic pattern) initially received during pre-exposure.

The infant-controlled habituation procedure (Horowitz, Paden, Bhana, \& Self, 1972) was designed to allow infants to control the length of each trial with their looking behavior. The session began with a control trial depicting a toy turtle and continued with four mandatory habituation trials. Trials began when infants visually fixated on the television monitor and terminated when infants looked away for 1.5 seconds or when 60 
seconds had elapsed. After the infants' attention decreased to a habituation criterion ( $50 \%$ reduction in visual fixation on two consecutive trials relative to their visual fixation on the first two trials) they received two no-change post-habituation trials. These additional trials were identical to the habituation trials and were presented to reduce the possibility of chance habituation. Following the post-habituation trials, infants received two unimodal auditory test trials depicting the familiar passage spoken in a novel prosody (change from approval to prohibition or vice versa). A final control trial depicting a toy turtle ended the testing session.

To make certain that infants were not overly fatigued, their visual fixations to the initial and final control trials were mathematically compared. Infants were judged as fatigued if their visual fixation to the final control trial was less than $35 \%$ of their fixation level to the initial control trial. In addition, approximately $58 \%$ of the infants were monitored by two observers and a Pearson product-moment correlation between the scores of the two observers served as our measure of inter-observer reliability. The Pearson product-moment correlation between the two observers averaged $.9971(S D=$. 0033).

\section{Predictions}

\section{Pre-exposure Session:}

It was predicted that there would be no significant differences in infants' total looking time during the pre-exposure session across the redundant audiovisual and the nonredundant auditory pre-exposure conditions because the pre-exposure displays only 
lasted a short period ( 60 seconds) and infants tend to look at displays for most of the available looking time.

\section{Habituation Session:}

Regarding my primary research question, it was predicted that infants would display significant visual recovery (increases in visual fixation from post-habituation looking to test trial looking) to a change in prosody in the unimodal auditory habituation session following redundant audiovisual but not unimodal auditory pre-exposure. These results were predicted given the hypothesis that redundant audiovisual speech as compared to unimodal auditory speech educates infants' attention to the amodal properties available in speech (such as affect, duration, tempo, rhythm, and intensity changes) throughout subsequent unimodal presentations. Moreover, it was predicted that infants in the redundant audiovisual pre-exposure condition would demonstrate significantly greater visual recovery to a change in prosody than infants in the unimodal auditory pre-exposure condition.

Given the vast literature indicating that infants prefer infant-directed speech conveying approval over prohibition, it was predicted that infants across pre-exposure conditions would selectively attend more to passages conveying approval over passages conveying prohibition. 


\section{Chapter IV}

Results

Pre-exposure Session

To determine if significant differences existed across pre-exposure conditions with respect to infants' total looking time during the pre-exposure session, an independent-sample t-test was conducted. The results revealed that the infants' interest in the pre-exposure event did not statistically differ across pre-exposure conditions, $t(38)=$ $0.97, p=.34$ (all tests two-tailed). These results indicate that infants across pre-exposure conditions displayed comparable interest in the pre-exposure event (redundant audiovisual pre-exposure event, $\mathrm{M}=56.20, S D=3.54$; unimodal auditory pre-exposure event, $\mathrm{M}=54.73, S D=5.75$ ), suggesting that the redundant audiovisual event was not more arousing than the unimodal auditory event.

\section{Habituation Session}

\section{Primary Analyses}

Visual recovery (increases in visual fixation from post-habituation looking to test trial looking) served as our primary dependent variable. Infants' visual recovery (and $S D$ ) to a novel prosody during the unimodal auditory habituation session as a function of pre-exposure condition (redundant audiovisual, unimodal auditory) are depicted in Figure 1.

To address the primary research question, whether three-and-a-half-month-olds were able to discriminate a change in prosody in the unimodal auditory habituation session, single-sample t-tests on infants' mean visual recovery score against the chance 
value of zero were conducted (see Figure 1). The results revealed that the infants in the redundant audiovisual pre-exposure condition demonstrated significant visual recovery to a change in prosody, $t(19)=2.56, p=.02$ (all tests two-tailed), whereas infants in the unimodal auditory pre-exposure condition did not demonstrate significant visual recovery to a change in prosody, $t(19)=-0.68, p=.51$. These results support predictions and indicate that three-and-a half-month-old infants were able to discriminate a change in prosody in the unimodal auditory habituation session following redundant audiovisual but not unimodal auditory pre-exposure to the amodal properties available in infant-directed speech.

To further evaluate the primary research question, whether pre-exposure condition, prosody type, and stimulus actress differentially effected infants' visual recovery scores, a three-way analysis of variance (ANOVA) was conducted on visual recovery with pre-exposure condition (redundant audiovisual, unimodal auditory), prosody type (approval, prohibition), and stimulus actress (actress 1, actress 2) as between subject factors. Results revealed a main effect of condition $(F(1,32)=5.41, p$ $=.03$ ), indicating that infants in the redundant audiovisual pre-exposure condition demonstrated greater visual recovery to a change in prosody than those infants in the unimodal auditory pre-exposure condition. These findings support predictions and indicate that discrimination of prosody is significantly greater following redundant audiovisual as compared with unimodal auditory pre-exposure.

Results of the three-way ANOVA and planned comparisons also revealed a significant pre-exposure condition by prosody type interaction $(F(1,32)=5.96, p=.02)$. 
This indicates that visual recovery for infants in the redundant audiovisual pre-exposure condition differed as a function of the prosody type (approval vs. prohibition) they received during the pre-exposure and habituation sessions but visual recovery for infants in the unimodal auditory pre-exposure condition did not differ as a function of prosody type (see Figure 2). Infants in the redundant audiovisual pre-exposure condition displayed greater visual recovery to a change in prosody if they were pre-exposed and habituated to phrases conveying approval as compared to phrases conveying prohibition. No other significant main effects or interactions (all $p \mathrm{~s}>.05$ ) were found.

To further explore the nature of the pre-exposure condition by prosody type interaction, single-sample t-tests were conducted on infants' mean visual recovery score against the chance value of zero for infants who received approval vs. prohibition during the unimodal auditory habituation session. Results (see Figure 2) revealed that the infants in the redundant audiovisual pre-exposure condition demonstrated significant visual recovery to a change in prosody following exposure to phrases conveying approval, $t(9)=2.74, p=.02$, but not following exposure to phrases conveying prohibition, $t(9)=0.76, p=.47$. In contrast, infants in the unimodal auditory preexposure condition did not demonstrate significant visual recovery to a change in prosody following exposure to phrases conveying approval $(t(9)=-1.74, p=.12)$ nor phrases conveying prohibition $(t(9)=0.58, p=.57)$. Taken together, these results indicate that only infants in the redundant audiovisual pre-exposure condition demonstrated significant visual recovery to a change in prosody when they were exposed to phrases conveying approval as compared to prohibition during the pre-exposure and habituation sessions. 


\section{$\underline{\text { Secondary Analyses }}$}

Secondary analyses were conducted to determine whether infants differentially attended more to phrases conveying approval vs. prohibition. Attention to phrases was measured by infants' total processing time and total number of looks away per minute during the unimodal auditory habituation session. Total processing time is defined as the total looking across all habituation and post-habituation trials and reflects infants' total interest in the unimodal auditory habituated event. Total number of looks away per minute is defined as the total amount infants disengage and look away per minute across all habituation and post-habituation trials and may reflect infants' avoidance of the stimulus event.

A two-way analysis of variance (ANOVA) on infants' total processing time during habituation with pre-exposure condition (redundant audiovisual, unimodal auditory) and prosody type (approval, prohibition) as between-subject factors was conducted. Analyses revealed a marginally significant main effect of prosody type $(F(1,36)=4.03, p=.052)$, indicating that infants across pre-exposure conditions visually fixated longer to one prosody type over the other (see Figure 3). During the habituation session, infants across pre-exposure conditions averaged 281.65 seconds $(S D=209.19)$ of total looking during phrases conveying approval, whereas they averaged 169.00 seconds $(S D=142.30)$ of total looking during phrases conveying prohibition. Thus, infants showed more overall interest in phrases conveying approval over phrases conveying prohibition. No other significant main effects or interactions (all $p \mathrm{~s}>.05$ ) were found. 
Further, a two-way analysis of variance (ANOVA) on infants' total number of looks away per minute during habituation with pre-exposure condition (redundant audiovisual, unimodal auditory) and prosody type (approval, prohibition) as betweensubject factors was conducted. Analyses revealed a significant main effect of prosody type $(F(1,36)=11.14, p=.002)$, indicating that infants across pre-exposure conditions looked away more often per minute from one prosody type over the other (see Figure 4). During the habituation session, infants across pre-exposure conditions averaged 7.01 (SD $=3.77$ ) looks away per minute during phrases conveying approval, whereas they averaged $13.51(S D=7.92)$ looks away per minute during phrases conveying prohibition. No other significant main effects or interactions (all $p \mathrm{~s}>.05$ ) were found.

These results converge with previous work indicating that young infants display greater responsiveness to infant-directed speech conveying approval as compared to prohibition (Fernald, 1993; Papousek et al.,1990). Taken together, the current findings suggest that infant-directed speech conveying approval as compared to prohibition differentially sustain attention and convey valence (for example, approach to approval and avoidance to prohibition). However, general preference for passages conveying positive over negative affect did not determine whether infants demonstrated significant visual recovery to a change in prosody. Only infants who received redundant audiovisual pre-exposure to phrases conveying approval as compared to prohibition demonstrated significant visual recovery to a change in prosody.

Secondary analyses were also conducted to determine whether the pre-exposure condition (redundant audiovisual vs. unimodal auditory) elicited different looking 
patterns in the unimodal auditory habituation session. A repeated measures analysis of variance (ANOVA) was performed with pre-exposure condition (redundant audiovisual vs. unimodal auditory) as the between-subjects factor and mean baseline looking, mean number of habituation trials, mean post-habituation looking, mean total processing time, and mean test looking as repeated measures to compare differences in looking patterns during habituation across pre-exposure conditions.

Despite the fact that the mean looking times were higher for the unimodal auditory than for the redundant audiovisual pre-exposure condition (see Table 1), analyses only revealed a marginally significant main effect of mean baseline looking $(F$ $(1,38)=4.07, p=.051)$. Infants in the unimodal auditory pre-exposure condition averaged 44.38 seconds $(S D=16.71)$ of looking during the first two trials of habituation, whereas infants in the redundant audiovisual pre-exposure condition averaged 32.50 seconds $(S D=20.36)$ of looking during the first two trials of habituation. The other measures did not approach significance (all $p \mathrm{~s}>.10$ ). Thus, infants in the redundant audiovisual and unimodal auditory pre-exposure condition differed marginally in their initial interest to the unimodal auditory habituation event (where infants in the unimodal auditory pre-exposure condition displayed greater initial interest than infants in the redundant audiovisual pre-exposure condition) but did not differ in their mean number of habituation trials, mean post-habituation looking, mean total processing time, or mean test looking. These analyses suggest that infants of both pre-exposure conditions showed similar patterns of visual fixation prior to and during test, indicating comparable levels of overall interest and final looking levels for the unimodal auditory habituation events. 
Taken together, these analyses indicate that on the basis of comparable looking patterns during the habituation session, infants in the redundant audiovisual pre-exposure condition as compared with infants in the unimodal auditory pre-exposure condition displayed strong visual recovery to a change in prosody. These results suggest infants across pre-exposure conditions (redundant audiovisual, unimodal auditory) may be attending to different aspects of the stimulus event. Infants in the redundant audiovisual pre-exposure condition may be tuned to the amodal properties available in the stimulus event, whereas infants in the unimodal auditory pre-exposure condition may be attending to other aspects of the stimulus event at the expense of attention to amodal properties. 


\section{Chapter V}

\section{Discussion}

Infants' first two years of life are filled with experiences which lay a foundation for language learning and production. It has been postulated that the exaggerated prosodic patterns characteristic of infant-directed speech have many advantages which aid infants in a multitude of perceptual tasks (Fernald, 1984). Most studies investigating infant-directed speech have been conducted on infants' perception of unimodal auditory speech (e.g., Moore et al., 1997; Spence \& Moore, 2003). However, research on audiovisual events indicates that intersensory redundancy in the form of temporal synchrony between auditory and visual stimulation recruits attention and facilitates perceptual learning of the amodal properties (e.g., affect, duration, patterns consisting of tempo, rhythm, and intensity changes) available in infant-directed speech more successfully than when the same information is presented unimodally to only one sensory modality (Bahrick \& Lickliter, 2000, 2002; Bahrick et al., 2007; Castellanos et al., 2004; Flom \& Bahrick, 2007). Given the benefits of intersensory redundancy, the effects of infant-directed speech for maintaining infants' attention and state, conveying affect and intention, aiding in parsing the speech stream, and facilitating learning of sound-meaning relations would likely be more pronounced in redundant audiovisual rather than in nonredundant (unimodal auditory, asynchronous audiovisual) contexts. Although, in early infancy, redundant audiovisual stimulation is more advantageous than unimodal auditory stimulation for directing attention to the amodal properties available in infantdirected speech, not all naturalistic events offer intersensory redundancy. 
Lickliter et al., (2006) posit that the detection of amodal properties in redundant audiovisual contexts serve to promote selective attention to those same stimulus properties in subsequent nonredundant contexts. In the present study, I assessed if amodal properties available in infant-directed speech could be detected in contexts where intersensory redundancy was not available following redundant audiovisual as compared with unimodal auditory pre-exposure.

Several important conclusions emerged from the findings of the present study. First, the present results demonstrate that redundant audiovisual pre-exposure differentially effects three-and-a-half-month old infants' detection of prosody in unimodal auditory speech. Infants who received redundant audiovisual as compared to unimodal auditory pre-exposure were able to subsequently attend to amodal properties in the unimodal auditory habituation and testing sessions. These results provide information about the contexts which guide selective attention to amodal properties. Intersensory redundancy initially serves to direct attention to amodal properties in infant-directed speech and attention to those properties is later extended to contexts where intersensory redundancy is not available (i.e., unimodal auditory stimulation).

Second, results reveal that prosodic patterns conveying approval as compared to prosodic patterns conveying prohibition were differentially detected. In the redundant audiovisual pre-exposure condition, infants who were exposed to phrases conveying approval demonstrated greater attention to a change in prosody than those infants who were exposed to phrases conveying prohibition. However, the same was not true for infants in the unimodal auditory pre-exposure condition. In the unimodal auditory pre- 
exposure condition, infants showed no visual recovery to a change in prosody and their attention did not differ as a function of the prosody type (approval vs. prohibition) they were exposed to. These results suggest that intersensory redundancy functions to facilitate the perception of prosody, highlighting intonation, affect, duration, tempo, rhythm, and the intensity that unites audible and visible speech.

Third, overall visual attention to the prosodic phrases during the habituation session was examined to determine if infants' total processing time and total number of looks away per minute was greater for phrases conveying approval vs. prohibition. Results indicate that infants of both pre-exposure conditions visually attended more during phrases conveying approval than phrases conveying prohibition and looked away more often per minute from phrases conveying prohibition than from phrases conveying approval. These results mirror findings from previous studies which indicate that infants prefer to listen to positive as compared to negative vocal affect (Fernald, 1993; Papousek et al.,1990). However, it should be noted that preferences for phrases conveying positive over negative affect did not determine whether infants demonstrated significant visual recovery to a change in prosody. Significant visual recovery scores were directly affected by infants' pre-exposure condition.

Lastly, analyses were conducted to determine whether the pre-exposure condition elicited differential looking during the unimodal auditory habituation session. Results revealed that infants of both pre-exposure conditions demonstrated similar patterns of visual interest in the stimulus events. On the basis of the same amount of pre-exposure stimulation $(56.20$ seconds $(S D=3.54)$ and 54.73 seconds $(S D=5.75)$ for infants in the 
redundant audiovisual and unimodal auditory pre-exposure conditions, respectively) and comparable looking patterns during habituation, infants who received redundant audiovisual pre-exposure displayed strong visual recovery to a change in prosody whereas infants who received unimodal auditory pre-exposure displayed no significant visual recovery to a change in prosody.

An alternative hypothesis has been proposed to explain why redundant audiovisual stimulation guides attention to amodal properties more successfully than unimodal auditory stimulation. This hypothesis posits that redundant audiovisual stimulation is more arousing and/or provides greater overall amount of stimulation. My data indicates that infants' looking to the events during the pre-exposure and habituation sessions did not statistically differ, providing no evidence that redundant audiovisual stimulation is more arousing than unimodal auditory stimulation. However, there may have been ceiling effects masking such differences as infants looked at the events $92 \%$ of the total available time.

It is also important to directly examine if temporal synchrony between auditory and visual stimulation is responsible for the enhanced detection of amodal properties, as predicted by the IRH, in subsequent unimodal stimulation by controlling for overall amount and type of stimulation. This can be accomplished by pre-exposing infants to asynchronous audiovisual stimulation, which offers the same amount and type of stimulation as synchronous audiovisual stimulation but eliminates intersensory redundancy, and examining if such exposure guides attention to amodal properties in subsequent unimodal auditory stimulation. It is predicted that if temporal synchrony 
between auditory and visual stimulation is important for guiding attention to amodal properties, then infants pre-exposed to asynchronous audiovisual stimulation (as compared to redundant audiovisual stimulation) should not demonstrate significant visual recovery to a change in prosody during unimodal auditory stimulation.

The present results provide added support for the notion of educating attention (Gibson, 1979) and suggest that intersensory redundancy available in audiovisual contexts serves as a bootstrapping mechanism that aids naïve perceivers to tune in to invariances across streams of stimulation. Redundant audiovisual contexts create the opportunity to educate attention or attune the perceptual system towards meaningful properties of the environment (e.g., amodal properties available in prosodic speech). Prior exposure to redundant audiovisual stimulation directs infants' attention to amodal properties available in prosodic speech and transfers to subsequent unimodal auditory stimulation. This suggests that detection of meaningful categories conveyed by prosody first emerges by detecting intersensory redundancy in audiovisual speech and is then generalized to unimodal auditory speech. Taken together with previous studies of precocial and human infants (Bahrick et al., 2002; Bahrick \& Lickliter, 2000, 2002; Bahrick et al., 2007; Castellanos et al., 2004; Castellanos et al., 2006; Flom \& Bahrick, 2007; Lickliter et al., 2002, 2004; Lickliter et al., 2006), these findings underscore the influence of intersensory redundancy on recruiting attention and facilitating perception of amodal properties during early infancy. 
Figure 1:

Infants' $(\mathrm{N}=40)$ mean visual recovery (and $S D$ ) to a novel prosody during the unimodal auditory habituation session as a function of pre-exposure condition (redundant audiovisual vs. unimodal auditory).

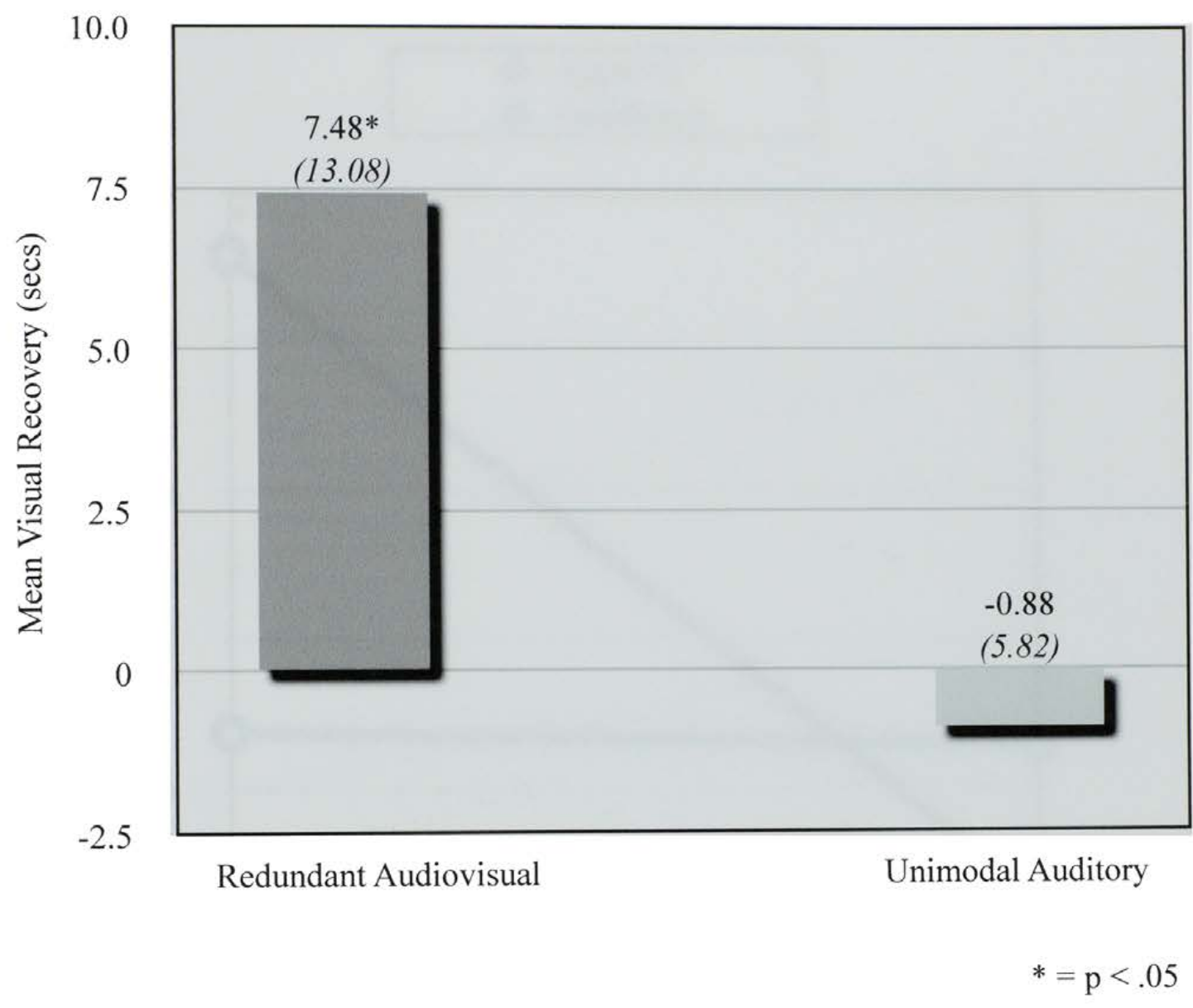


Figure 2:

Infants' mean visual recovery to a novel prosody during the unimodal auditory habituation session as a function of pre-exposure condition (redundant audiovisual vs. unimodal auditory) and prosody type (approval vs. prohibition).

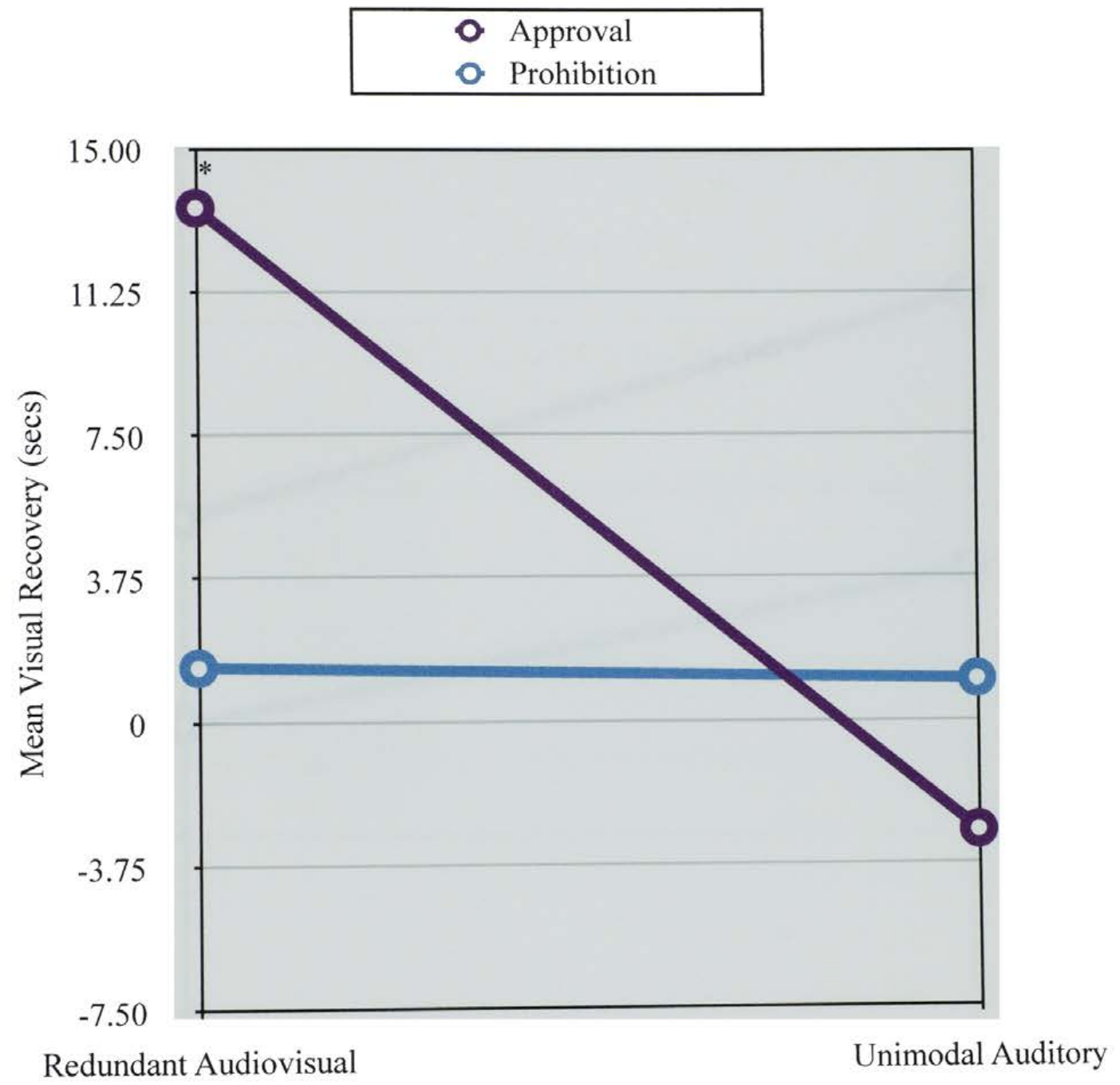

Pre-Exposure Condition

$*=\mathrm{p}<.05$ 
Figure 3:

Infants' mean total processing time during the unimodal auditory habituation session as a function of pre-exposure condition (redundant audiovisual vs. unimodal auditory) and prosody type (approval vs. prohibition).

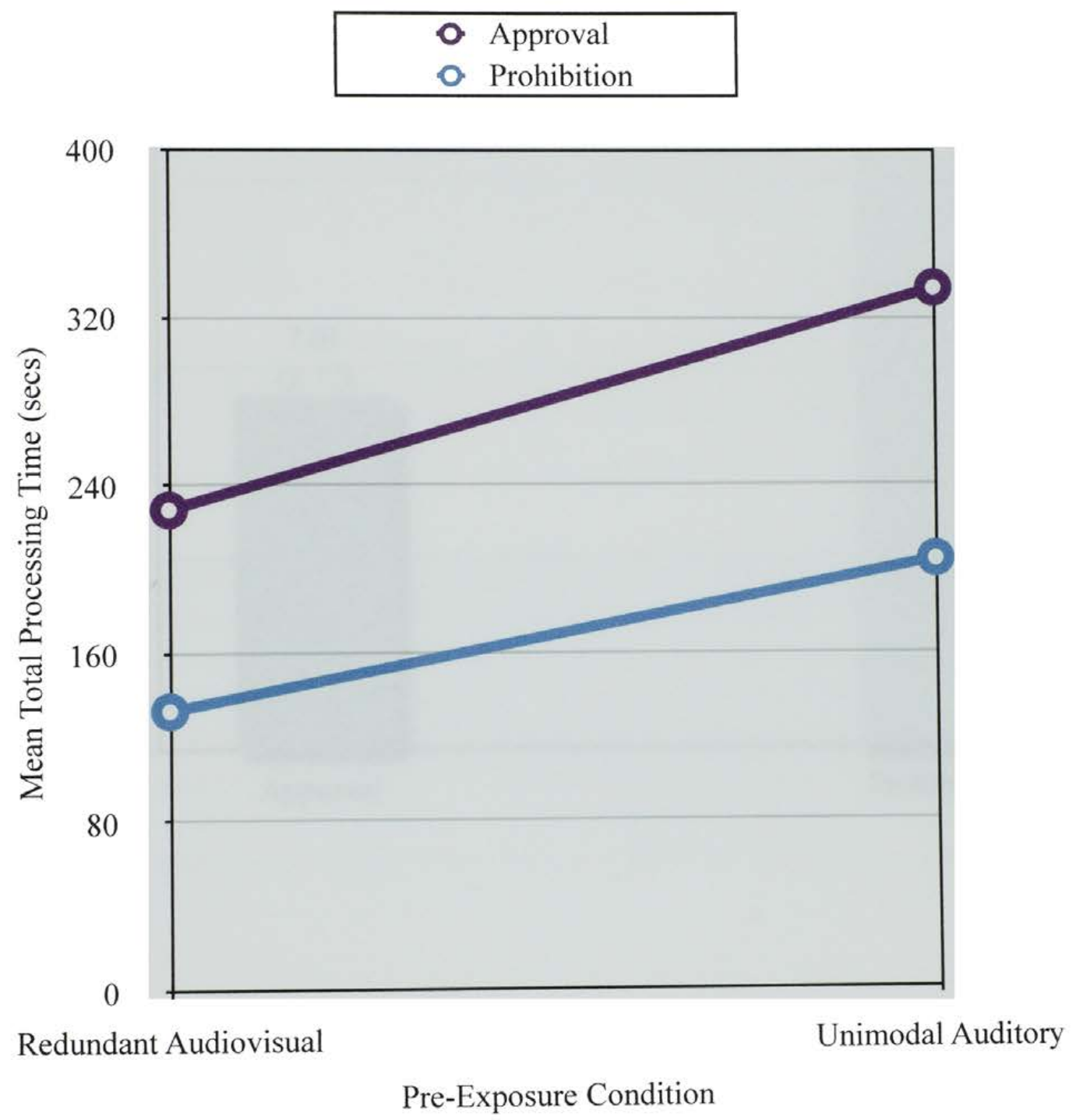


Figure 4:

Infants' $(\mathrm{N}=40)$ mean total number of looks away (and $S D$ ) per minute during the unimodal auditory habituation session as a function of prosody type (approval vs. prohibition).

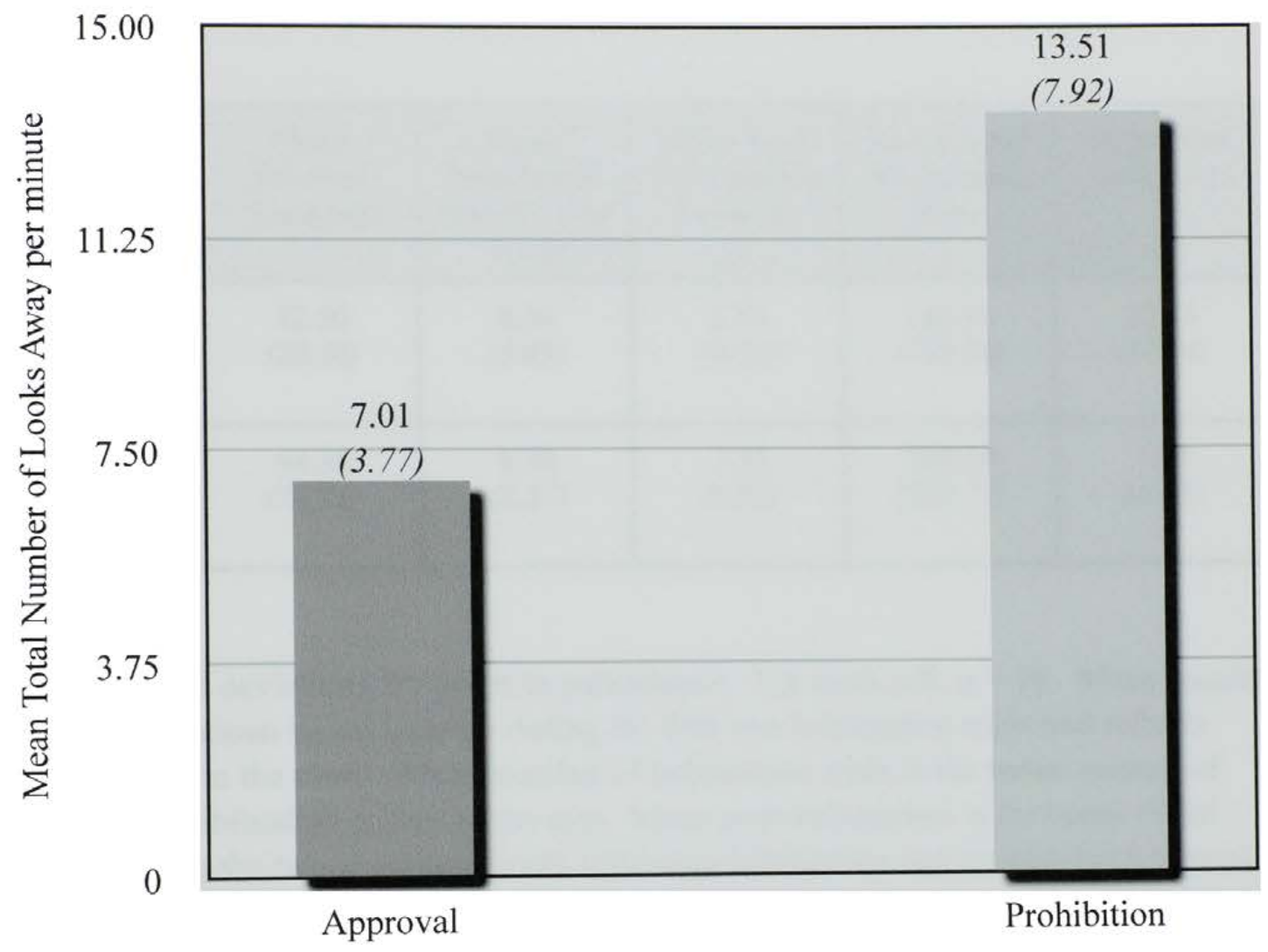




\section{Table 1:}

Means (and $S D s$ ) for visual fixation in seconds during the unimodal auditory habituation session: mean baseline looking, mean number of habituation trials, mean post-habituation looking, mean total processing time, and mean test looking as a function of pre-exposure condition.

\begin{tabular}{|c|c|c|c|c|c|}
\hline $\begin{array}{c}\text { Pre- } \\
\text { Conposure }\end{array}$ & $\begin{array}{c}\text { Mean } \\
\text { Baseline } \\
\text { Looking }\end{array}$ & $\begin{array}{c}\text { Mean } \\
\text { Number of } \\
\text { Habituation } \\
\text { Trials }\end{array}$ & $\begin{array}{c}\text { Mean Post- } \\
\text { Habituation } \\
\text { Looking }\end{array}$ & $\begin{array}{c}\text { Mean Total } \\
\text { Processing } \\
\text { Time }\end{array}$ & $\begin{array}{c}\text { Mean Test } \\
\text { Looking }\end{array}$ \\
\hline $\begin{array}{c}\text { Redundant } \\
\text { Audiovisual }\end{array}$ & $\begin{array}{c}32.50 \\
(20.36)\end{array}$ & $\begin{array}{c}8.30 \\
(3.05)\end{array}$ & $\begin{array}{c}5.14 \\
(4.22)\end{array}$ & $\begin{array}{c}180.95 \\
(158.24)\end{array}$ & $\begin{array}{c}12.63 \\
(14.74)\end{array}$ \\
\hline $\begin{array}{c}\text { Unimodal } \\
\text { Auditory }\end{array}$ & $\begin{array}{c}44.38 \\
(16.71)\end{array}$ & $\begin{array}{c}8.70 \\
(3.37)\end{array}$ & $\begin{array}{c}7.36 \\
(6.02)\end{array}$ & $\begin{array}{c}269.70 \\
(203.71)\end{array}$ & $\begin{array}{c}7.08 \\
(3.65)\end{array}$ \\
\hline
\end{tabular}

Note: Standard deviations are given in parentheses. For each cell, $\underline{\mathbf{n}}=20$. Mean baseline looking is the mean visual fixation during the first two habituation trials and reflects initial interest in the event. Mean number of habituation trials is the mean amount of trials before habituation criterion was met. Mean post-habituation is the mean visual fixation during the two no-change trials following habituation and reflects final interest in the event. Mean total processing time is the mean total looking across all habituation and post-habituation trials and reflects total interest in the habituated event. Mean test looking is the mean visual fixation during the two test trials depicting a novel prosody. 
Apgar, V. (1953). A proposal for a new method of evaluation of the newborn infant. Current Researches in Anesthesia and Analgesia, 32, 260-267.

Bahrick, L. E. (1983). Infants' perception of substance and temporal synchrony in multimodal events. Infant Behavior and Development, 6, 429-451.

Bahrick, L. E. (1987). Infants' intermodal perception of two levels of temporal structure in natural events. Infant Behavior and Development, 10, 387-416.

Bahrick, L. E. (1988). Intermodal learning in infancy: Learning on the basis of two kinds of invariant relations in audible and visible events. Child Development, 59, 197-209.

Bahrick, L. E. (2001). Increasing specificity in perceptual development: Infants' detection of nested levels of multimodal stimulation. Journal of Experimental Child Psychology, 79, 253-270.

Bahrick, L. E., Flom, R., \& Lickliter, R. (2002). Intersensory redundancy facilitates discrimination of tempo in 3-month-old infants. Developmental Psychobiology, $41,352-363$.

Bahrick, L. E., \& Lickliter, R. (2000). Intersensory redundancy guides attentional selectivity and perceptual learning in infancy. Developmental Psychology, 36, 190-201.

Bahrick, L. E., \& Lickliter, R. (2002). Intersensory redundancy guides early perceptual and cognitive development. In: R. V. Kail (Ed.), Advances in child development and behavior, Vol. 30 (pp. 153-187). New York: Academic Press.

Bahrick, L. E., Lickliter, R., \& Flom, R. (2006). Up versus down: The role of intersensory redundancy in the development of infants' sensitivity to the orientation of moving objects. Infancy, 9, 73-96.

Bahrick, L. E., Shuman, M., \& Castellanos, I. (2007). Intersensory redundancy facilitates infants' perception of meaning in speech passages. Manuscript in preparation.

Castellanos, I., Shuman, M. A., \& Bahrick, L. E. (2004, May). Intersensory redundancy facilitates infants' perception of meaning in speech passages. Poster presented at the International Conference on Infant Studies, Chicago, IL. 
Castellanos, I., Vaillant-Molina, M., Lickliter, R., \& Bahrick, L. E. (2006, October). Intersensory redundancy educates infants' attention to amodal information during early development. Poster presented at the International Society for Developmental Psychobiology, Atlanta, GA.

Cooper, R. P., \& Aslin, R. N. (1990). Preference for infant-directed speech in the first month after birth. Child Development, 61, 1584-1595.

Christophe, A., Gout, A., Peperkamp, S., \& Morgan, J. (2003). Discovering words in the continuous speech stream: The role of prosody. Journal of Phonetics, 31 , 585-598.

Darwin, C. (1877). A biographical sketch of an infant. Mind, 2, 285-294.

Fernald, A. (1984). The perceptual and affective salience of mothers' speech to infants. In L. Feagans, C. Garvey, \& R. Golinkoff (Eds.), The origins and growth of communication (pp. 5-29). Norwood, NJ: Ablex.

Fernald, A. (1985). Four-month-old infants prefer to listen to motherese. Infant Behavior and Development, 8, 181-195.

Fernald, A. (1989). Intonation and communicative intent in mothers' speech to infants: Is the melody the message? Child Development, 60, 1497-1510.

Fernald, A. (1993). Approval and disapproval: Infant responsiveness to vocal affect in familiar and unfamiliar languages. Child Development, 64, 657-674.

Fernald, A., \& Kuhl, P. K. (1987). Acoustic determinants of infant preference for motherese speech. Infant Behavior and Development, 10, 279-293.

Fernald, A., \& Mazzie, C. (1991). Prosody and focus in speech to infants and adults. Developmental Psychology, 27, 209-221.

Fernald, A., Taeschner, T., Dunn, J., \& Papousek, M. (1989). A cross-language study of prosodic modifications in mothers' and fathers' speech to preverbal infants. Journal of Child Language, 16, 477-501.

Flom, R., \& Bahrick, L. E. (2007). The development of infant discrimination of affect in multimodal and unimodal stimulation: The role of intersensory redundancy. Developmental Psychology, 43, 238-252. 
Gerken, L., \& Aslin, R. N. (2005). Thirty years of research on infant speech perception: The legacy of Peter W. Jusczyk. Language Learning and Development, 1, 5-21.

Gibson, J.J. (1979). The ecological approach to visual perception. Hillsdale, NJ: Erlbaum.

Gogate, L. J., \& Bahrick, L. E. (1998). Intersensory redundancy facilitates learning of arbitrary relations between vowel sounds and objects in seven-month-old infants. Journal of Experimental Child Psychology, 69, 1-17.

Gogate, L. J., Bahrick, L. E., \& Watson, J. D. (2000). A study of multimodal motherse: The role of temporal synchrony between verbal labels and gestures. Child Development, $71,878-894$.

Grieser, D. L. \& Kuhl, P. K. (1988). Maternal speech to infants in a tonal language: Support for universal prosodic features in motherese. Developmental Psychology, $24,14-20$.

Hamann, S. B., Squire, L. R., \& Schacter, D. L. (1995). Perceptual thresholds and priming in amnesia. Neuropsychology, 9, 3-15.

Hirsh-Pasek, K., Kemler Nelson, D. G., Jusczyk, P. W., Cassidy, K. W., Druss, B., \& Kennedy, L. (1987). Clauses are perceptual units for young infants. Cognition, 26, 269-286.

Hollich, G., Newman, R. S., \& Jusczyk, P. W. (2005). Infants' use of synchronized visual information to separate streams of speech. Child Development, 76, 598-613.

Horowitz, F. D., Paden, L., Bhana, K., \& Self, P. (1972). An infant-control procedure for studying infant visual fixations. Developmental Psychology, 7, 90.

Jusczyk, P. W., Hirsch-Pasek, K., Kemler Nelson, D. G., \& Kennedy, L. J. (1992). Perception of acoustic correlates of major phrasal units by young infants. Cognitive Psychology, 24, 252-293.

Lickliter, R., Bahrick, L. E., \& Honeycutt, H. (2002). Intersensory redundancy facilitates prenatal perceptual learning in bobwhite quail embryos. Developmental Psychology, 38, 15-23.

Lickliter, R., Bahrick, L. E., \& Honeycutt, H. (2004). lntersensory redundancy enhances memory in bobwhite quail embryos. Infancy, 5, 253-269. 
Lickliter, R., Bahrick, L. E., \& Markham, R. G. (2006). Intersensory redundancy educates selective attention in bobwhite quail embryos. Developmental Science, 9 , 604-615.

Lewkowicz, D. J. (1992). Infants' responsiveness to the auditory and visual attributes of a sounding/moving stimulus. Perception and Psychophysics, 52, 519-528.

Lewkowicz, D. J. (1996). Infants' response to the audible and visible properties of the human face. I: Role of lexical-syntactic content, temporal synchrony, gender, and manner of speech. Developmental Psychology, 32, 347-366.

Mandel, D. R., Kemler Nelson, D. G., \& Jusczyk, P. W. (1996). Infants remember the order of words in a spoken sentence. Cognitive Development, 11, 181-196.

Moore, D. D., Spence, M. J., \& Katz, G. S. (1997). Six-month-olds' categorization of natural infant-directed utterances. Developmental Psychology, 33, 980-989.

Morgan, J. L. (1996). Prosody and the roots of parsing. Language \& Cognitive Processes, 11, 69-106.

Nazzi, T., Kemler Nelson, D. G., Jusczyk, P. W., \& Jusczyk, A. M. (2000). Sixmonth-olds' detection of clauses embedded in continuous speech: Effects of prosodic well-formedness. Infancy, 1, 123-147

Pickens, J., Field, T., Nawrocki, T., \& Martinez, A. (1994). Full-term and preterm infants' perception of face-voice synchrony. Infant Behavior and Development, 17, 447-455.

Papousek, M., Bornstein, M. H., Nuzzo, C., Papousek, H., \& Symmes, D. (1990). Infant responses to prototypical melodic contours in parental speech. Infant Behavior and Development, 13, 539-545.

Papousek, M., Papousek, H., \& Symmes, D. (1991). The meanings of melodies in motherese in tone and stress languages. Infant Behavior and Development, 14, 415-440.

Schacter, D. L. (1987). Implicit Memory: History and Current Status. Journal of Experimental Psychology, 13, 501-518.

Schacter, D., \& Badgaiyan, R. D. (2001). Neuroimaging of Priming: New Perspectives on Implicit and Explicit Memory. Current Directions in Psychological Science, 10, 1-4. 
Shafer, M. J., Shucard, D. W., \& Jaeger, J. J. (1999). Electrophysiological indices of cerebral specialization and the role of prosody in language acquisition in threemonth-old infants. Developmental Neuropsychology, 15, 73-109.

Stern, D. N., Spieker, S., Barnett, R. K., \& MacKain, K. (1983). The prosody of maternal speech: Infant age and context related changes. Journal of Child Language, 10, $1-15$.

Stern, D. N., Spieker, S., \& MacKain, K. (1982). Intonation contours as signals in maternal speech to prelinguistic infants. Developmental Psychology, 18, 727-735.

Spence, M. J., \& Moore, D. S. (2003). Categorization of infant-directed speech: Development from 4 to 6-months. Developmental Psychobiology, 42, 97-109.

Trainor, L. J., Austin, C. M., \& Desjardins, R. N. (2000). Is infant-directed speech prosody a result of the vocal expression of emotion? Psychological Science, 11, 188-195.

Tulving, E., \& Schacter, D. L. (1990). Priming and human memory systems. Science, 247,301-306.

Vaillant-Molina, M., Gutierrez, M. E., \& Bahrick, L.E., (2005, November). Infant memory for modality-specific properties of contingent and noncontingent events: The role of intersensory redundancy in self perception. Poster presented at the Society for Developmental Psychobiology, Washington, D. C.

Walker-Andrews, A. (1997). Infants' perception of expressive behaviors: Differentiation of multimodal information. Psychological Bulletin, 121, 437-456.

Werker, J. F., \& McLeod, P. J. (1989). Infant preferences for both male and female infant-directed talk: A developmental study of attentional and affective responsiveness. Canadian Journal of Psychology, 43, 230-246. 